Apidologie, 1972, 3 (4), 309-340.

\title{
DAS POLLENSPEKTRUM VON HONIGEN AUS DEN ITALIENISCHEN ALPEN
}

\author{
Le spectre pollinique de quelques miels \\ provenant des Alpes italiennes
}

\section{Günther VORWOHL}

Universität Hohenheim

Landesanstalt für Bienenkunde

\author{
SUMMARY \\ THE POLLENSPECTRUM OF SOME HONEYS FROM THE ITALIEN ALPS
}

The pollen grains in the sediment of 23 authentic honey samples collected in the Italien Alps were identified so closely as possible at present. The results are tabulated. The following characteristic pollen-combination was found :

Castanea sativa, Myosotis, Fraxinus ornus, Pirus-group, Lotus, Erica carnea, Rhododendron hirsutum/ferrugineum, Salix, Ranunculus acer-group, Cruciferae R, Rubus-group, Melilotus, Trifolium repens-group, Trifolium pratense-group, Helianthemum Umbelliferae, especially $\mathbf{H}$ (Heracleum and others), Labiatae M, Labiatae S, Compositae T, Rumex, Caryophyllaceae, Papaver, Prunus-group, Rhinanthus, Plantago, Campanula, Compositae J. Gramineae.

Alpine and submediterranean elements are found in the same honey. This seems to be typical for honeys from the southern slope of the Alp mountains. The pollenspectra are very rich. In the average 65 different pollen forms were found per honey. Some pollen species not sufficiently described in melissopalynological literature are photographed.

\section{ZUSAMMENFASSUNG}

Die Pollen im Sediment von 23 authentischen Honigen aus den italienischen Alpen wurden so genau wie zur Zeit möglich identifiziert. Die Ergebnisse sind in tabellarischer Form dargestellt. Es ergibt sich die folgende charakteristische Pollenkombination :

Castanea sativa, Myosotis, Fraxinus ornus, Pirus-Gruppe, Lotus, Erica carnea, Rhododendron hirsutum/ferrugineum, Salix, Ranunculus acer-Gruppe, Cruciferen R, Rubus-Gruppe, 
Melilotus, Trifolium repens-Gruppe, Trifolium pratense-Gruppe, Helianthemum Umbelliferen, insbesondere H (Heracleum u.a.), Labiaten M, Labiaten S, Compositen T, Rumex, Caryophyllaceen, Papaver, Prunus-Gruppe, Rhinanthus, Plantago, Campanula, Composite J, Gramineen.

Typisch ist das gleichzeitige Auftreten von alpinen und submediterranen Elementen. Diese Kombination dürfte nur in den Honigen vom Südrand der Alpen vorkommen. Die Pollenspektren sind sehr artenreich (im Durchschnitt 65 unterscheidbare Formen pro Honig). Einige bisher im Rahmen der Melissopalynologie unzureichend beschriebene Pollen sind abgebildet.

Vor der Deutschen Botanischen Gesellschaft wurde 1968 ein erster Bericht über die italienischen Honige gegeben (VorwoHL, 1969). Inzwischen konnte weiteres Material beschafft und untersucht werden. Hier soll zunächst das mikroskopische Bild der Honige aus einem Teil des italienischen Alpengebiets detailliert beschrieben werden, das sich gut charakterisieren und von dem der übrigen Honige Italiens abgrenzen läßt.

\section{MATERIAL UND METHODEN}

Untersucht wurden 23 Honige, 22 aus der Region Alto Adige-Trentino und 1 aus dem alpinen Teil der Region Veneto. Die nördlichste und gleichzeitig östlichste Probe stammt aus dem äußersten Valle Aurina (Ahrntal). Im Westen geht das Einzugsgebiet bis Meran und im Süden bis Rovereto und Riva del Garda. Wir verdanken das Material Herrn G. Piana, Castel San Pietro, der auch die nötigen zusätzlichen Angaben beschaffte (Ort, an dem der Honig erzeugt wurde, Höhenlage, Zeitpunkt der Schleuderung des Honigs, Betriebsweise, Wanderung, mutmaßlich genutzte Tracht).

Die Anfertigung der Präparate und die Auswertung erfolgte nach den Angaben der Internationalen Kommission für Bienenbotanik (LouveauX, Maurizio, Vonwohl, 1970/71). Je ein Präparat pro Honig (10 g) wurde vollständig Bahn für Bahn durchgesehen. Ausgezählt wurden mindestens 200 Pollenkörner, also die zur Bestimmung der Häufigkeitsklassen nötige Zahl.

\section{ERGEBNISSE}

Die identifizierten Pollenformen sind in Tabelle 1 niedergelegt. Diese gibt auch Auskunft über die Häufigkeit, mit der die jeweiligen Formen gefunden wurden. Senkrecht gelesen bringt die Tabelle alle Pollen, die in einer Honigprobe gefunden wurden, horizontal gelesen erlaubt sie die Feststellung, in wieviel der untersuchten Proben eine bestimmt Pollenform vorkommt.

\section{CHARAKTERISIERUNG DER EINZELNEN HONIGE}

Nr. 1, AT, (J 49), Predoi (1 300-1 600 m), Valle Aurina (Nebental des Pustertals), imkerliche Trachtangabe : Alpenwiesen, Alpenrosen, 7/1967, Wanderimkerei :

Es handelt sich um einen typischen alpinen Blütenhonig. Im Pollenbild dominiert Myosotis. Auf die Herkunft aus montaner, wiesenreicher Lage 
weist auch das Vorkommen von Rhinanthus-Pollen hin. Myosotis und Rhinantus gibt es auch im Flachland. Aus melissopalynologischer Sicht kann man aber, zumal in Italien, von montan alpinen Elementen sprechen.

Nr. 2, AT, (J 52), Campo Tures, 1800 m, nördlich des Pustertals; imkerliche Trachtangabe : Alpenrosen, 7/1967, Wanderimkerei :

Es liegt ein alpiner Blütenhonig (Rhododendron, Myosotis, Rhinanthus) vor. Andeutungsweise finden sich auch Pollen der tieferen Lagen (Ailanthus und Castanea sativa). Entweder flogen die Bienen größere Strecken talwärts oder die Pollen entstammen Honigresten, die bei der Wanderung in tiefere Lagen eingetragen wurden.

Die imkerlichen Trachtangaben stimmen nur teilweise. Auch wenn man Myosotis bei der Zählung außer acht läßt, machen die Ericaceen, Rhododendron und Erica carnea, nur ca. $6 \%$ des Pollens aus. Der Honig stammt vielmehr aus gemischter Tracht : Trifolium repens-Gruppe, Rhinanthus-Gruppe, RubusGruppe, Myosotis, Erica carnea, Rhododendron. Das Pollenspektrum umfaßt 43 Formen.

Nr. 3, AT, (J 58), Brunico (Bruneck) Pustertal, 1 400-1 $700 \mathrm{~m}$; imkerliche Trachtangaben : Esparsette, Salbei, 7/1967, Wanderimkerei : Der Honig stammt aus vielseitiger Tracht : Obst, Weißklee-Gruppe, Korbblütler 'T, Schneeheide, Vergißmeinnicht, Robinie.

Die imkerlichen Angaben stimmen nur in beschränktem Umfang, Esparsette und Salbeipollen kommen mit Häufigkeiten von weniger als $1 \%$ vor. Auch wenn man in Rechnung setzt, daß der Salbei stark unterrepräsentiert ist und eine Auszählung unter Vernachlässigung von Myosotis vornimmt, kommt man auf einen Nektaranteil des Salbeis von bestenfalls wenigen Prozenten.

Das Pollenspektrum vereinigt in sich alpine und submediterrane Elemente : Erica carnea, Rhododendron, ergänzt durch Myosotis, aus den tieferen, wärmeren Lagen stammen Fraxinus ornus und Morus. Artenreiches Pollenspektrum : 74 Formen.

Nr. 4, AT, (J 55), Brunico (Bruneck), Pustertal, 600 m, imkerliche Trachtangabe : Robinie, Löwenzahn, Wiesen, 6/1967, Wanderimkerei : Die botanische Herkunft des Honigs ist vielseitig. Neben dem nach imkerlichen Angaben zu erwartenden Anteil aus Robinie und Wiesentracht (Esparsette, WeißkleeGruppe, Vergißmeinnicht) und dem nur geringfügig vertretenen Löwenzahn (Composite T) machen sich alpine Ericaceen und Obst stärker bemerkbar. Das Spektrum umfaßt neben den alpinen Elementen (Erica carnea, Rhododendron, Crocus, dazu Larix) auch submediterrane Elemente (Morus, Fraxinus ornus). Aus Gartenanlagen stammen wohl vereinzelte, als Palmenpollen identifizierte Einfalter. Artenreiches Pollenspektrum : 73 Formen. 
Nr. 5, Ve, (J 48), Campolongo (Paß, östlich von Bozen, südlich Brunico, nördlich des Marmolada-Massivs), 1 700-2 000 m, 8/1967, Wanderimkerei :

Der Höhenlage und den imkerlichen Angaben entsprechend stammt der Honig vorwiegend von alpinen Ericaceen. Doch fehlen submediterrane Elemente nicht ganz (Fraxinus ornus, Morus). Anzahl der unterschiedenen Pollenformen : 49.

Nr. 6, AT, (J 51), Valle Sarentina (nördlich Bozen), 1200 m, imkerliche Trachtangaben : Alpenrose, Heidelbeere, Wald, 7/1967, Wanderimkerei :

Der Honig stammt aus vielseitiger Tracht, der mikroskopische Befund stimmt weitgehend mit den imkerlichen Angaben überein. Honigtau-Elemente fehlen praktisch ganz. Die Angabe Wald stimmt also nur insofern, als die Edelkastanie zum Honig beigetragen hat, die neben Nektar auch Honigtau liefert, der allerdings oft arm an Pilzelementen und Algen ist und dann mikroskopisch nur wenig in Erscheinung tritt.

Neben den alpinen Elementen Rhododendron und Erica carnea (ergänzt durch Myosotis und Rhinanthus) findet sich Fraxinus ornus. Auf die tieferen Lagen mit Weinklima verweist das Auftreten von Vitis. Das Pollenspektrum weist 47 Pollenformen auf.

Nr. 7, AT, (J 50), Bolzano (Bozen), $1200 \mathrm{~m}$, imkerliche Trachtangaben : Kornblume, Skabiose, Salbei, Himbeere, Tannenhonigtau, 7/1967, Wanderimkerei :

Es handelt sich um einen Honigtauhonig mit Blütenanteilen aus Kornblumen- (Composite C) und Rubus-Tracht. Salbei (Labiaten S) und Skabiosen (Dipsacaceen, Knautia-Gruppe) ließen sich nur vereinzelt nachweisen, sind allerdings auch unterrepräsentiert. Die alpine Konbination ist vertreten mit Erica carnea, Rhododendron, Myosotis und Rhinanthus. Als submediterranes Element tritt wiederum Fraxinus ornus auf. Das Pollenspektrum umfaßt 59 Formen.

Nr. 8, AT, (J 47), Merano, 300-500 m, imkerliche Trachtangaben : Obst, Wiesen, Wald, 7/1967, Wanderimkerei :

Entsprechend den imkerlichen Angaben handelt es sich um einen Honigtau-Blüten-Mischhonig, bei dem sich die Edelkastanie mikroskopisch stark in den Vordergrund drängt. Die Wiesenflora ist besonders durch Salbei vertreten. Die alpinen Elemente treten entsprechend der Höhenlage zahlenmäßig stark zurück, sind aber noch nachweisbar (Erica carnea, Rhododendron, Myosotis). Fraxinus ornus und Chamaerops (wohl aus Gartenanlagen) zeigen den mediterran-submediterranen Einfluß an. 63 Pollenformen wurden unterschieden.

Nr. 9, AT, (J 53), Merano, 1900 m, inkerliche Trachtangaben : Alpenrose, Alpenflora, 7/1967, Wanderimkerei : 
Der Honig stammt aus vielseitiger Tracht. Neben den alpinen Elementen Erica carnea, Rhododendron, sowie Myosotis und Rhinanthus machen sich auch Trachtpflanzen der tieferen Lagen bemerkbar : Castanea sativa, Fraxinus ornus. Das Pollenspektrum umfaßt 61 Pollenformen.

Nr. 10, AT, (J 57), Castelbello (mittleres Venosta-Tal), 1100 m, imkerliche Trachtangaben : Himbeere, Heidelbeere, Alpenrose, Tannenhonigtau, $7 / 1967$ :

Es handelt sich um einen Blütenhonig mit Honigtauanteil. Das Pollenspektrum ist weit gefächert und umfaßt Elemente aus allen Vegetationsstufen : Rhododendron, Erica carnea, Myosotis, Rhinanthus Castanea sativa, Vitis Fraxinus ornus, Cistus. Die imkerlichen Trachtangaben stimmen, abgesehen von dem kleinen Edelkastanienanteil, gut mit dem mikroskopischen Befund überein. 70 Pollenformen wurden unterschieden.

Nr. 11, AT, (J 75), Alta Val di Non (nördlich Trient), 1967 :

Der Honig stammt aus vielseitiger Blütentracht (Obst, Esparsette, Klee) und vorwiegend aus den niedrigen Lagen. Die alpinen Ericaceen sind nur noch vereinzelt $\mathrm{zu}$ finden, desgleichen Myosotis und Rhinanthus. Hingegen erreicht Fraxinus ornus die Häufigkeit des Begleitpollens. Das Pollenspektrum umfaßt 53 Pollenformen.

Nr. 12, AT, (J 68), Val di Fiemme (nordöstlich Trient), imkerliche Trachtangaben : Wiesen, 1967 :

Blütenhonig mit Honigtauanteil, bei dem die Edelkastanie eine mindestens ebenso große Rolle spielt wie die Wiesentracht. Die alpinen Elemente sind noch vorhanden, treten aber in den Hintergrund. Neben Fraxinus ornus zeigt Cercis den submediterranen Einfluß an. Extrem reiches Pollenspektrum : 93 unterscheidbare Pollenformen.

Nr. 13, AT, (J 69), Canal S. Bovo (östlich von Trient, nahe der Grenze Venetiens), 1967 :

Der Honig zeigt vorwiegend den Charakter eines alpinen Blütenhonigs : Rhododendron, Erica carnea, Rhinanthus, Myosotis, Gentiana, Crocus, Pedicularis comosa-Gruppe. Vereinzelt ist jedoch auch Fraxinus ornus vorhanden. 53 Pollenformen wurden unterschieden.

Nr. 14, AT, (J 74), Val di Sole (westlich Bozen), 1967 :

Es handelt sich um einen weitgehend alpin bestimmten Blütenhonig. Wie bei Nr. 13 auch hier sehr vereinzelt Pollen der Pedicularis comosa-Gruppe. Sehr vereinzelt finden sich aber auch Fraxinus ornus und Chamaerops. Das Pollenspektrum umfaßt 52 Formen.

Nr. 15, AT, (J 71), Bassa Val di Non (nordwestlich Trient vor der Mündung des Noce in die Etsch), 1967 : 
Der einzige Honig in dieser Reihe, in dem Fraxinus ornus den Leitpollen stellt. Vereinzelt ist auch “ Sulla » vertreten (Hedysarum coronarium), die in den Honigen des südlichen und mittleren Italiens eine große Rolle spielt. Die alpinen Pollen fehlen jedoch nicht ganz. Über die Bedeutung der Schmuckoder Manna-Esche als Trachtpflanze für die Bienen liegen mir keine Angaben vor, insbesondere, ob sie Nektar liefert oder nicht. Zum mindesten scheint sie ein außerordentlich ergiebiger und beliebter Pollenspender zu sein. Reiches Pollenspektrum mit 74 Formen.

Nr. 16, AT, (J 63), Trento, imkerliche Trachtangaben : gemischte Waldtracht, 1967 :

Es handelt sich um einen Blüten-Honigtau-Mischhonig mit größerem Edelkastanienanteil. Ein schwacher alpiner Einschlag läßt sich noch nachweisen (Erica carnea, Rhododendron, Myosotis, Rhinanthus). Submediterran sind Morus, Fraxinus ornus und Cotinus coggygria. Das Pollenspektrum ist sehr reich, es umfaßt 82 Formen.

Nr. 17, AT, (J 65), Lomaso (westlich Trient), imkerliche Trachtangaben : gemischte Waldtracht, 1967 :

Die Probe ist als Blüten-Honigtau-Mischhonig zu kennzeichnen, wobei der Blütenanteil von einer Vielzahl von Pflanzen stammt. Es finden sich sowohl alpine als auch mediterrane Elemente (vereinzelt auch Myrtaceen). Sehr reiches Spektrum : 82 Pollenformen.

Nr. 18, AT, (J 66), Val Rendena (westlich Trient, nördlich Tione di Trento), imkerliche Trachtangaben : Wiesen, 1967 :

Es handelt sich um einen Edelkastanien-Honigtau-Mischhonig mit alpinen und mediterranen Elementen (Fraxinus ornus, Erica arborea, Citrus, Cotinus coggygria). Pollen von Wiesenpflanzen sind vorhanden, bestimmen aber nicht das Bild des Honigs. 60 Pollenformen konnten unterschieden werden.

Nr. 19, AT, (J 72), Val Sugana (oberes Brentatal, östlich Trient), 1967 :

Edelkastanienhonig mit Honigtau-Anteil ohne deutliche alpine Elemente, Fraxinus ornus und Ceratonia zeigen den submediterranen-mediterranen Einfluß an. Das reiche Pollenspektrum umfaßt 79 Formen.

Nr. 20, AT, (J 64), Vezzano (bei Trient), imkerliche Trachtangaben : gemischte Waldtracht, 1967 :

Es handelt sich um einen Blüten-Honigtau-Mischhonig, dessen Pollenspektrum gleichermaßen alpine und submediterrane Einschläge zeigt (erstmals auch Olea). Reiches Pollenspektrum mit 76 Formen.

Nr. 21, AT, (J 62), Rovereto (südlich Trient), imkerliche Trachtangaben : gemischte Waldtracht, 1967 :

Der Honig stammt aus gemischter Blüten- und Honigtau-Tracht. Die 
alpinen Elemente treten wenig hervor (Erica carnea, Myosotis, Rhinanthus). Submediterrane-mediterrane Elemente sind : Hedysarum coronarium, Cotinus coggygria (knapp Begleitpollen), Fraxinus ornus. Interessant ist das Auftreten von Thesium (Taf. II, Abb. 5 a u. b, eine Trockenrasen-Pflanze, aus der Familie der Santalaceen). 56 Pollenformen wurden unterschieden.

Nr. 22, AT (J 67), Terragnolo (östlich Rovereto), imkerliche Trachtangaben : Wald, 1967 :

In Übereinstimmung mit den imkerlichen Angaben liegt ein Honigtauhonig vor. Das Pollenspektrum umfaßt sowohl alpine als auch submediterrane Elemente (Sulla, Olea, Ceratonia, Erica arborea, Fraxinus ornus). Reiches Pollenspektrum : 70 Formen.

Nr. 23, AT, (J 73), Riva del Garda (Ufer des Gardasees), 1967 : Es handelt sich um einen Edelkastanienhonig mit alpinen und submediterranen Pollenformen : sehr reiches Spektrum; 80 Formen.

Das Pollenspektrum der südtiroler Honige kann als hochcharakteristisch gelten und dürfte sich in dieser Ausprägung nur in Honigen vom Südrande der Alpen finden. Stellt man die Pollen zusammen, die in $3 / 4$ des untersuchten Materials vertreten sind, so ergibt sich die folgende Kombination :

Castanea sativa, Myosotis, Fraxinus ornus, diese Pollen können die Häufigkeit eines Leitpollen erreichen. Ferner gehören zur Kombination Pollen der Pirus-Gruppe, Lotus, Erica carnea und Rhododendron hirsutum/ferrugineum. Die genannten Formen erreichen im vorliegenden Material die Häufigkeit des Begleitpollens. Als wichtige Einzelpollen wurden mit großer Regelmäßigkeit gefunden : Salix, Ranunculus acer-Gruppe, Cruciferen R, Rubus-Gruppe, Melilotus, Trifolium repens-Gruppe, Trifolium pratense-Gruppe, Helianthemum Umbelliferen, insbesondere $\mathrm{H}$ (Heracleum und andere), Labiaten M, Labiaten S, Compositen T. Ferner waren als Einzelpollen in $3 / 4$ des Probenmaterials vertreten : Rumex, Caryophyllaceen, Papaver, Prunus-Gruppe, Rhinanthus, Plantago, Campanula, Composite $\mathbf{J}$, Gramineen. Charakteristisch ist das gleichzeitige Vorkommen von Pollen alpiner und submediterraner Pflanzen. Lediglich in einem Honig (Nr. 19) aus dem oberen Brentatal fehlen die alpinen Elemente ganz, und nur der nördlichste der Honige (Nr. 1) erweist sich als rein alpin. Diese hochspezifische Kombination ergibt sich aus dem Relief des Landes, in dem die verschiedenen Höhenstufen luftlinienmäßig eng aneinander gerückt sind. Die Mehrzahl der Proben stammt aus Imkereien, die ihre Völker zur besseren Nutzung des Nektar - und Honigtau-Angebots des Gebiets verstellen (sogenannte Wanderung), wodurch sich die Pollen der Flora der verschiedenen Wanderplätze überlagern können. Das sehr regelmäßige Auftreten alpiner Elemente weist aber darauf hin, daß die Bienen die hohen Lagen offensichtlich aktiv aufsuchen, denn das Anwandern dieser Plätze ist oft technisch unmöglich. 
Das gerade aufgezählte Spektrum wird ergänzt durch weitere alpine, submediterrane und gelegentlich auch mediterrane Elemente, die schon bei der Kurzbeschreibung der Honige aufgeführt wurden.

Auffallend ist der große Reichtum der Spektren. Im Durchschnitt konnten 65 Pollenfomen unterschieden werden. Bei der Beurteilung dieser Zahl ist in Rechnung zu setzen, daß eine c vollständige ) Durchsicht eines Präparats vorgenommen wurde, wodurch auch Pollen erfaßt werden, deren Häufigkeit nur Bruchteile eines Promills erreicht. Bei Routineuntersuchungen für praktische Zwecke kann und muß man sich mit mehr oder minder gründlichen, ( orientierenden » Analysen begnügen. Der Artenreichtum beruht vorwiegend auf vereinzelt vorkommenden Formen. Im Durchschnitt finden sich pro Honig nur 3,6 Pollenformen in den drei ersten Häufigkeitsklassen (LP, BP, wi. EP).

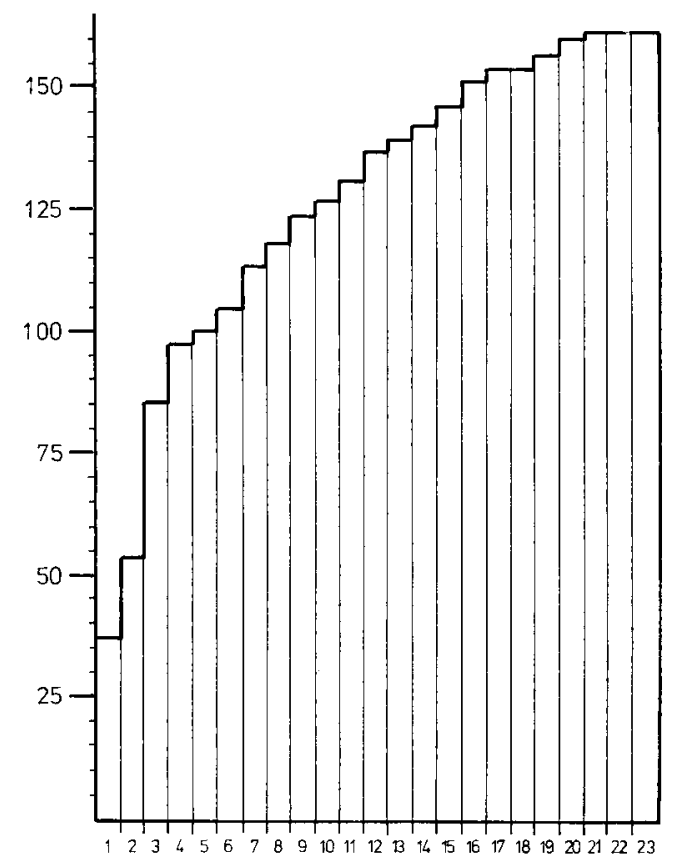

FIG. 1. - Courbe cumulative (type GuinocheT) représentant en abscisses le nombre des échantillons analysés et en ordonnées le nombre des formes polliniques identifiées. La forme de la courbe indique qu'une nouvelle augmentation du nombre des échantillons n'entraîne plus une augmentation sensible du nombre des espèces rencontrées.

Aвв. 1. - Kumulationskurve (Typ GuinocheT) der mit wachsender Probenzahl gefundenen Pollenarten (Erläuterung siehe Text). Ordinate : Zahl der gefundenen Pollenarten, Abszisse : laufende Nummer der Honige.

Man darf annehmen, daß die 23 untersuchten Honige bei der angewandten, sehr eingehenden Durchsicht ausreichen, um sich eine Übersicht über das Pollenspektrum der Honige des Alto Adige-Trentino zu verschaffen. Zur 
Kontrolle wurde eine Kumulationskurve vom Typ Guinochet gezeichnet. Als Unterlage diente die Tabelle 1, in der die Honige entsprechend den Fundorten von Norden nach Süden fortschreitend angeordnet sind. Es wurde jeweils ausgezählt, wieviel Pollenformen vorkommen, die in den vorangegangenen Honigen noch nicht gefunden wurden. Die Kumulationskurve beginnt ein Plateau aufzuzeigen, woraus man entnehmen kann, daß beim gegebenen Grad der Kenntnisse das Formenpotential der südtiroler Honige annähernd ausgeschöpft ist.

Die Zahl der Pollen, die nicht identifiziert werden konnten, war gering und blieb bei der Auszählung unter $1 \%$.

Maurizio hat sich bereits 1958 eingehend mit den Honigen der italienischen Schweiz beschäftigt. Wie zu erwarten, ist das Ergebnis ähnlich, aber nicht völlig gleich. Auffallend ist besonders, daß Fraxinus ornus nicht erwähnt wird. Auch Obst wird nicht als regelmäßiger Bestandteil erwähnt. Letzteres ist verständlich, da der Obstbau in Südtirol eine größere Rolle spielt als im Tessin. Nicht gefunden haben wir den Pollen der Mehlprimel. Auch hinsichtlich der Häufigkeit der einzelnen Pollenarten bestehen Unterschiede, sowohl was die Regelmäßigkeit des Auftretens im Material als auch die Häufigkeit der einzelnen Formen angeht. Dafür kann aber auch die unterschiedliche Methode bei der Darstellung der Ergebnisse verantwortlich sein, die einen Vergleich bis ins Detail nicht zuläßt. Z.B. scheinen in den südtiroler Honigen Tilia, Polygonum bistorta und Campanula seltener zu sein als in den Honigen des Tessins.

Soweit ich sehe, wurde bisher der Pollen von Thesium erstmals in Honig nachgewiesen. Er ist aber wohl zu selten, als daß er eine größere Rolle bei der Herkunftsbestimmung spielen könnte.

Bei der praktischen Anwendung der Melissopalynologie handelt es sich oft darum, eine geographische Herkunftsbezeichnung zu überprüfen oder die geographische Herkunft einer nicht gekennzeichneten Probe festzustellen. Von einem Honig, der als südtiroler Produkt bezeichnet wird, ist zu erwarten, daß er einen großen Teil der oben aufgezählten charakteristischen Kombination von alpinen und submediterranen/mediterranen Pollen enthält. Weit weniger häufig ist mit rein alpinen Honigen zu rechnen und mit Honigen, denen alpine Elemente fehlen. Diese letzteren zeigen dann die Pollenkombination des nichtalpinen Italiens (Vorwohl, 1969, Battaglini-Bernardini und Ricciar delli-d'Albore, 1971).

Bei ausreichender Übung und Erfahrung wird man bei Honigen mit südalpiner Kombination zwischen tessiner und südtiroler Honigen unterscheiden können. Ủber andere südalpine Honige (Piemont, Friaul) liegen noch keine Veröffentlichungen vor, die Ansatzpunkte für detaillierte Differenzierungen bieten. 
Die rein alpinen Honige Südtirols lassen sich natürlich nicht von entsprechenden Honigen der anderen Alpenländer unterscheiden.

Frau Dr. A. Fossel überließ mir freundlicherweise Protokolle über 27 von ihr analysierten Honigen zur Auswertung. Die mikroskopische Durchsicht umfaßte eine orientierende Analyse und die Schätzung der Pollenhäufigkeit. 6 der untersuchten Honige stammen aus Südtirol, 21 aus Südkärnten. Bei den letzteren interessierte die Frage, ob sie auch das oben beschriebene südalpine Pollenbild zeigen.

Die Honige aus Südtirol stammen alle aus großen Höhenlagen (1 $400 \mathrm{~m}$ und höher), entsprechend dominiert der alpine Charakter (Ericaceen, Myosotis und andere Wiesenflora). Trotzdem findet sich in drei Fällen der Pollen der Edelkastanie aus tieferen Lagen. Zusätzlich zu den in Tabelle 1 aufgeführten Pollen wurde noch Pollen der Colchicum- und Atropa-Gruppe gefunden. Das “ Plateau » der Guinochet-Kurve ändert sich also durch das Hinzutreten von sechs weiteren Proben nur unwesentlich.

Die Honige aus Südkärnten zeigen in ihrem Spektrum bereits merkliche Abweichungen von den Honigen des Alto Adige-Trentino. Recht regelmäßig (in jeweils 15 von 21 Fällen) kommen vor :

Myosotis (maximal als Leitpollen), Obst (bis zur Häufigkeit des Begleitpollens), Cruciferen (maximal als Begleitpollen), Rubus (maximal als Begleitpollen), Trifolium repens-Gruppe (maximal als Leitpollen), Trifolium pratense-Gruppe (maximal als Begleitpollen), Umbelliferen $(\mathrm{A}+\mathrm{H})$, Compositen $\mathrm{T}$ (maximal als Leitpollen).

Der Pollen der Edelkastanie kommt in den 21 Honigen aus Südkärnten nicht vor, ebenso wurde Fraxinus ornus nicht gefunden. Diese Pollen sind also zum mindesten in Honigen Südkärntens selten. Die alpinen Elemente treten stark zurück, kein Pollen der alpinen Kombination (von Myosotis abgesehen) ist regelmößig vertreten. Bemerkenswert ist das Vorkommen von Loranthus europaeus in zwei Fällen. Dieser Pollen wurde in den südtiroler Honigen bisher nicht gefunden (wohl aber in Honigen aus dem Apennin, Vorwoнц, 1969). Mediterrane Elemente finden sich erwartungsgemäß nicht.

\section{BESCHREIBUNG EINIGER POLLENARTEN}

Über einige der in der vorliegenden Untersuchung gefundenen Pollen liegen nur unzureichende Beschreibungen in melissopalynologischer Literatur vor, weshalb sie hier näher behandelt werden.

1. - Ceratonia siliqua L., Leguminosae, Caesalpinoideae, Rosales. Pollenprobe : Bot. Garten Hohenheim 1971, Präparat Hohenheim 1971, 
Tafel I, Abb. la - lf. Verbreitung : Persien, Kleinasien, östliches Mittelmeergebiet, im westlichen Mediterraneum kultiviert. Ziemlich kleine, blaß graue isopolare PK. Polansicht abgerundet quadrangulär, bzw. abgerundet fünfseitig, Seitenansicht circular bis breit oval, 4 oder 5 Porenfalten, Pore nicht bei allen PK gut ausgebildet, Faltenlänge $19 \mu$, Abstand der Faltenränder voneinander $7-8 \mu$, Faltenenden spitz, Faltenränder gerade. Endopore breiter als die Exopore (falls ausgebildet). Exine $1,3 \mu$ dick, Intime $1 \mu$, an den Keimstellen verstärkt. Exine fein und gleichmäßig reticuliert. Das Lumen der Brochi hat weniger als $1 \mu$ Durchmesser. $\mathbf{P}=27,2 \mu, \mathbf{A}=27,4 \mu,(n=50)$. Die Ähnlichkeit mit Pollen von Vertretern der Gattung Citrus ist auffällig, CitrusPollen ist allerdings im Durchschnitt größer; immerhin kommt z.B. C. aurantium (Tafel I, Abb. 2a) auch in der Größe Ceratonia nahe. Zudem gibt es bei Citrus-Pollen meist eine sehr starke Variation der Pollengröße, bedingt durch viele abortive PK. Das Reticulum ist bei Citrus durchweg großmaschiger. Das Lumen der Brochi von Ceratonia erscheint immer rundlich, bei Citrus hingegen unregelmäßiger, auch ist das Reticulum bei Citrus immer mehr oder minder heterobrochat (Tafel I, Abb. 2a-2c).

\section{2. - Cercis siliquastrum L., Leguminosae, Caesalpinioideae, Rosales.}

Pollenprobe Ricciardelli d'Albore, Perugia 1970, Präparat Hohenheim 1970. Tafel II, Abb. 3a - 3c. Verbreitung : Kleinasien, Krim, Mittelmeergebiet, als Zierbaum kultiviert. Ziemlich kleine blaß graugebliche isopolare PK. Polansicht abgerundet triangulär. Seitenansicht breit oval. 3 Falten mit schwach ausgeprägter Poroide. Falten spitz zulaufend, Faltenränder gerade, am ungefärbten PK kaum sichtbar. Abstand der Faltenenden voneinander ca. $8 \mu$, Faltenlänge ca. $19 \mu$, Breite maximal $7 \mu$, Sporoderm dünn, Exine $0,7 \mu$, Intime $0,7 \mu$. Gleichmäßiges feines Reticulum, Durchmesser des Lumen unter $1 \mu$. Keine Margo. $\mathbf{P}=25,4 \mu, \mathrm{A}=26,4 \mu,(n=50)$.

\section{3. - Cotinus coggygria Scop., Anacardiaceae, Sapindales.}

Pollenprobe und Präparat Bologna 1970. Verbreitung : Mediterraneum bis China, Zierstrauch. Kleine, gelbliche isopolare PK. Polansicht circulär, Seitenansicht breit oval. 3 Porenfalten, Länge der Falte $18 \mu$, Abstand der Faltenenden voneinander $3-4 \mu$. Falten schmal, Enden mäßig spitz. Exopore rund, Durchmesser $3-4 \mu$. Endopore breiter, undeutlich begrenzt. Exine $1,5 \mu$ dick, Intine $1 \mu$, Exine an den Poren verdickt. Exine striat-reticulat. $\mathrm{P}=21,3 \mu, \mathrm{A}=20,7 \mu(n=50)$. 


\section{DANKSAGUNG}

Die Untersuchung erfolgte im Rahmen des Programms “" Vergleichende palynologische Untersuchung der Honige des Mittelmeergebiets", das freundlicherweise von der Deutschen Forschungsgemeinschaft gefördert wird, wofür ich auch an dieser Stelle danken möchte.

\section{RÉSUME}

Les pollens contenus dans le sédiment de 23 miels authentiques provenant des Alpes italiennes ont été soumis à un examen complet. Les échantillons proviennent de la région du Haut Adige Trentin et des Alpes de Vénitie. Les pollens qui ont été trouvés figurent dans le tableau 1. Une courte description des différents échantillons donne pour chacun d'eux l’origine géographique précise et les particularités mélisso-palynologiques.

Le spectre pollinique caractéristique des miels étudiés est fourni par la liste suivante :

Castanea sativa, Myosotis, Fraxinus ornus (peuvent atteindre le stade de pollens dominants), Groupe Pirus, Lotus, Erica carnea, Rhododendron hirsutum/ferrugineum, (peuvent atteindre dans le matériel étudié le stade de pollens d'accompagnement), Salix, Groupe Ranunculus acer, Crucifères R, Groupe Rubus, Melilotus, Groupe Trifolium repens, Groupe Trifolium pratense, Ombellifères, particulièrement $H$ (Heracleum entre autres), Labiées $M$, Labiées $S$, Composées T, (avec la fréquence de pollens isolés importants), Rumex, Caryophyllacées, Papaver, Groupe Prunus, Rhinanthus, Plantago, Campanula, Composées J, Graminées.

Un caractère typique est la présence simultanée de pollens de plantes alpines et subméditerranéennes qui est en rapport avec le relief du pays qui fait que des étages différents sont très voisins à vol d'oiseau.

Les spectres sont riches en espèces (en moyenne 65 formes polliniques différenciables par miel). Une courbe cumulative du type Guinochet montre que le potentiel des formes polliniques présentes est pratiquement épuisé. Les formes non identifiées restent toujours, dans les comptages au-dessous de $1 \%$.

Le spectre pollinique des miels du Tirol du sud est semblable à celui des miels du Tessin étudiés par Maurizio. Il manque toutefois à ces derniers le pollen de Fraxinus ornus. Les miels de la Carinthie du sud sont déjà très différents de ceux des Alpes italiennes.

Le pollen de Ceratonia siliqua est décrit et comparé à celui des Citrus. Les pollens de Cercis siliquastrum, Cotinus coggygria et Thesium sp. sont représentés.

\section{LITERATUR}

Allen M. Y., 1937, European Bee Plants and their Pollen. Bee Kingdom League, Alexandria. Armbruster L., Jacobs J., 1934/35, Pollenformen und Honigherkunftsbestimmung. Arch. Bienenkde., 15, (8), 277-308 u. 16, (1, 2/3), 17-106.

Armbruster L., CEnike G., 1929, Die Pollenformen als Mittel zur Honigherkunftsbestimmung. Wachholtz, Neumünster.

Baroni E., 1969, Guida botanica d'Italia. Capelli, Bologna.

Battaglini-Bernardini Marcella, Ricciardelli d'Albore G., 1971, Contributo alla conoscenza dei mieli italiani. I. Origine botanica dei mieli della Provincia di Perugia. Apicoltore Moderno, 62, (4), 64-82. 
Beug H. J., 1961, Leitfaden der Pollenbestimmung. 1. Lfg., G. Fischer, Stuttgart.

Erdtman G., 1966, Pollen morphology and plant taxonomy. I Angiosperms. Hafner, New York.

Evenius J., Focke Elida, 1967, Mikroskopische Untersuchung des Honigs. In Handbuch der Lebensmittelchemie. Bd. 5, Teil 1, 560-590, Springer, Berlin.

Fiori A., 1969, Nuova flora analytica d'Italia. Edagricole, Bologna.

Fossel Annemarie, Rutrner F., 1966, Die kleine Wachsblume, Cerinthe minor L., eine charakteristische Trachtpflanze paunonischer und alpiner Trockengebiete. Z. Bienenforsch., 8, (5), 168-177.

Grieber C., 1930/31, Zur Pollenanalyse des Honigs. Z. Unters. Lebensmittel, 59, (1), 63-79, (2/3), 197-211, (5), 441-471, 61, (3), 241-306.

Hazsurzzki B., 1952, Qualitative u. quantitative Untersuchung ungarischer Robinienhonige. Mitt. Ungar. Akad. Wiss. Biol. Sekt. I, (3), 317-417. Ungarisch, deutsche Zusammenfassung.

Hegr, 1969, Illustrierte Flora von Mitteleuropa. 2. Auflage, hrsg. v. D. Hartl, Bd. 6/1, Hanser, München.

Hodges Dorothy, 1952, The pollen loads of the honeybee. Bee Research Association Ltd., London.

Honowitz A., BAUm B., 1967, The arboreal pollen flora of Israel. Pol . Spor., 9, 71-93.

Hyde H. A., Adams K. F., 1958, An atlas of airborne pollen grains. Macmillan, London.

Louveaux J., 1970, Atlas photographique d'analyse pollinique des miels. Service de la répression des fraudes et du contrôle de la qualité, Paris.

Louveaux J., Maurizio Anna, Vonwohl G., 1970, Methodik der Melissopalynologie. Apidologie, 1, (2), 193-209.

Maurizio Anna, 1958, Tipi di mieli della Svizzera italiana. Riv. Svizz. Apicolt., 41, (1), 20-28.

Maurizio Anna, Louveaux J., 1965, Pollen de plantes mellifères d'Europe. Union des groupements apicoles français, Paris.

Pitschmann H., Reisigl H., Schiechtr H., 1965, Flora der Südalpen. Fischer, Stuttgart.

Vorwoнl G., 1968, Das Pollenbild der italienischen Honige. Ber. Botan. Ges., 81, (11), $512-527$.

Vorwoнl G., 1968, Grundzüge einer modernen Pollenbeschreibung im Rahmen der Bienen-und Honigkunde. Z. Bienenforsch., 9, (5), 224-230.

ZANDER E., 1935, 1937, 1941, 1949, 1951, Beiträge zur Herkunftsbestimmung bei Honig. Bd. 1 Reichsfachgruppe Imker, Berlin, Bd. 2 u. 3 Liedloff, Loth u. Michaelis, Leipzig, IV Ehrenwirth, München.

Anschrift des Verfassers : Dr. G. VonwohL, Universität Hohenheim. D 7 Stuttgart 70 Emil-WoLfF-Str. 60, BRD. 
Aвв. 1. - Ceratonia siliqua : a. Pollen in der Pollage, Focus 1, 1000 x.; b. Pallen in der Seitenlage, Aufsicht auf das Intercolpium, Focus 1, 1000 x.; c. Pollen in der Pollage, optischer äquatorialer Schnitt, 4 Keimstellen, 1000 x.; d. Seitenlage, Aufsicht auf die Porenfalte, Focus 2, 1000 x.; e. Pollage, optischer äquatorialer Schnitt, 5 Keimstellen, 1000 x.; f. Seitenlage, optischer meridionaler Schnitt.

Aвв. 2. - Citrus spp. : a. C. aurantium, Pollen in Pollage, Focus 1,1000 x.; b. C. limetta, Pollen in Seitenlage, Aufsicht auf das Intercolpium, Focus 2,1000 x.; c. C. limetta, Seitenlage, Aufsicht auf das Intercolpium und Porenfalte, Focus 1, $1000 \mathrm{x}$.

\section{PLANCHE I}

FIg. 1. - Ceratonia siliqua : a. Vue polaire, Foc. 1.1000 x.; b. Vue méridienne, intercolpium, Foc. 1.1000 x.; c. Vue polaire, coupe équatoriale, 4 apertures. 1000 x.; d. Vue méridienne, aperture, Foc. 2.1000 x.; e. Vue polaire, coupe optique équatoriale, 5 apertures 1000 x.; f. Coupe optique méridienne.

Fig. 2. - Citrus sp. : a. C. aurantium, vue polaire, Foc. 1.1000 x.; b. C. limetta, vue méridienne, intercolpium, Foc. 2.1000 x.; c. C. limetta, vue méridienne, intercolpium et aperture. Foc. $1.1000 x$. 

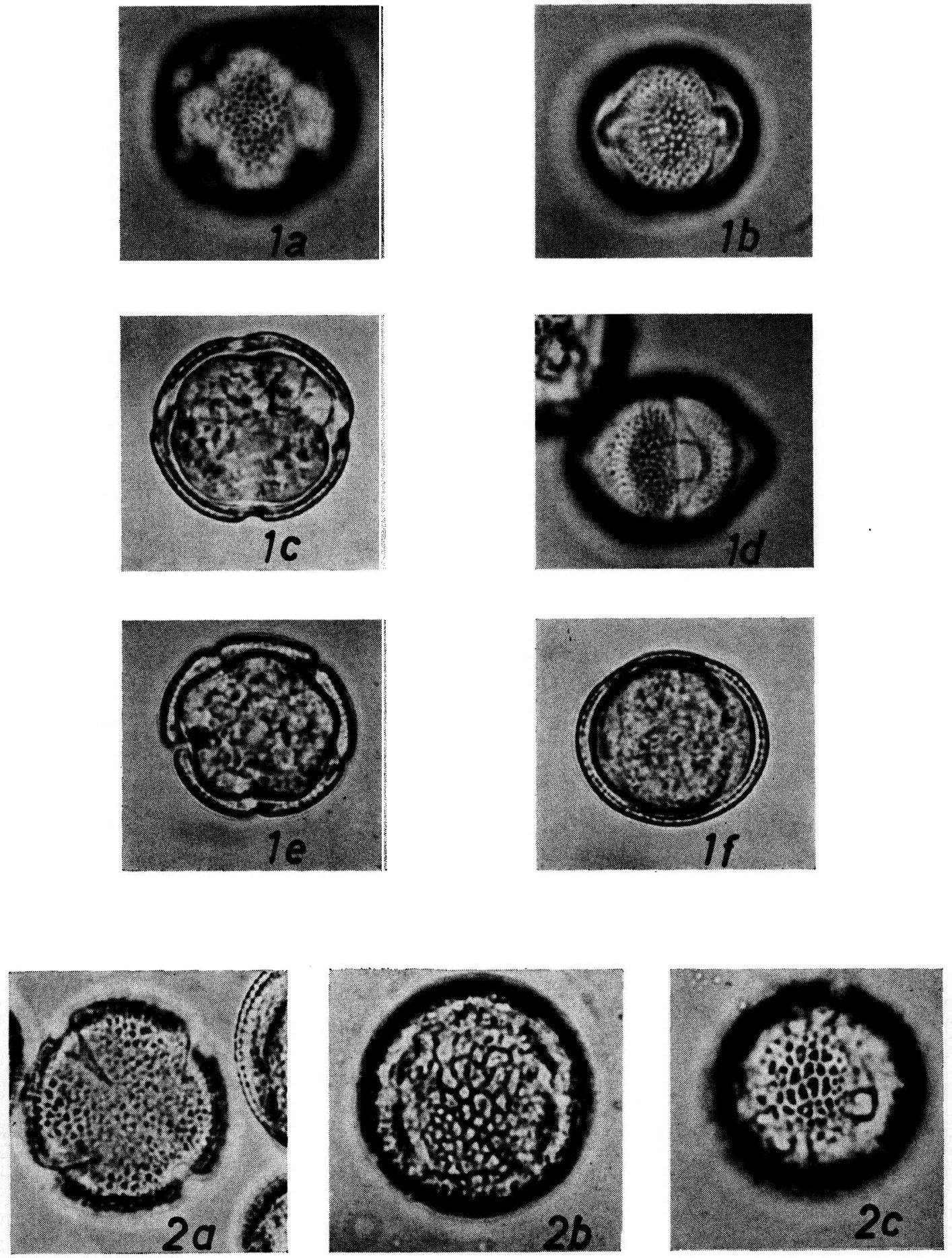
Авв. 3. - Cercis siliquastrum : a. Pollen in Pollage, optischer äquatorialer Schnitt, 1000 x.; b. Pollage, Aufsicht auf das Polfeld, Focus 1, 1000 x. Präparat mit Fuchsin gefärbt; c. Pollen in der Seitenlage, optischer meridionaler Schnitt, $1000 \mathrm{x}$.

Aвв. 4. - Cotinus coggygria : a. Pollen in der Pollage, Aufsicht auf das Polfeld, Focus 1, 1000 x.; b. Pollage, optischer äquatorialer Schnitt, 1000 x; c. Pollen in Seitenlage, optischer, meridionaler Schnitt, 1000 x.; d. wie 4 c.; e. Seitenlage, Aufsicht auf Porenfalte und Intercolpium.

Focus 2.1000 x.; f. Seitenlage, Aufsicht aufdie Porenfalte, Focus 1, 1000 x.

Авв. 5. - Thesium spec. : a Pollen von Thesium im Sediment von Honig AT 21, Focusierung auf das Reticulum, 1000 x.; b Gleiches PK wie in $5 a$, Focusierung auf die trichotomosulcate Keimstelle, $1000 \mathrm{x}$, in der Umgebung des Pollenkorns feine Kristalle, sogenannte “ kristalline Masse ».

\section{PLANCHE II}

Frg. 3. - Cercis siliquastrum : a. Vue polaire, coupe optique équatoriale. $1000 \mathrm{x}$. b. Vue polaire, aire polaire, Foc. 1. 1000 x. Coloration fuchsine.; c. Vue méridienne, coupe optique méridienne. $1000 \mathrm{x}$.

FIg. 4. - Cotinus coggygria : a. Vue polaire, aire polaire, Foc. 1. 1000 x.; b. Vue polaire, coupe optique équatoriale. $1000 \mathrm{x}$; c. Coupe optique méridienne $1000 \mathrm{x}$; d. Même chose que 4 c.; e. Vue méridienne, aperture et intercolpium. Foc. 2. $1000 \mathrm{x}$; f. Vue méridienne, aperture. Foc. 1. 1000 x.

Fig. 5. - Thesium sp : a. Pollen de Thesium dans le sédiment du miel AT 21; mise au point sur le réticule. $1000 \mathrm{x}$; b. Même grain de pollen que 5 a. Mise au point sur l'aperture trichotomosulquée 1000 x. Autour du grain de pollen on note la présence d'une masse finement cristalline. 

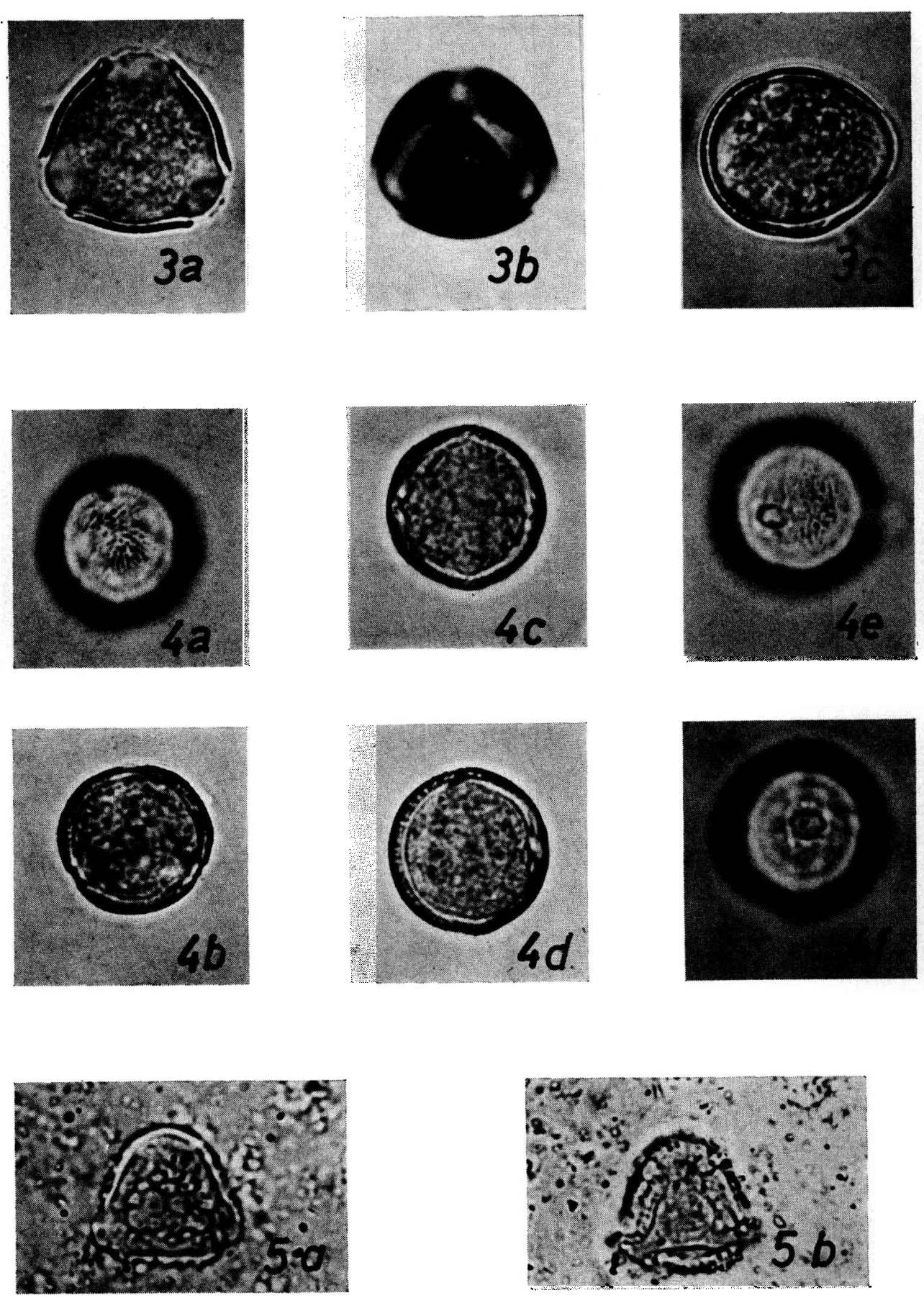
G. VORWOHL

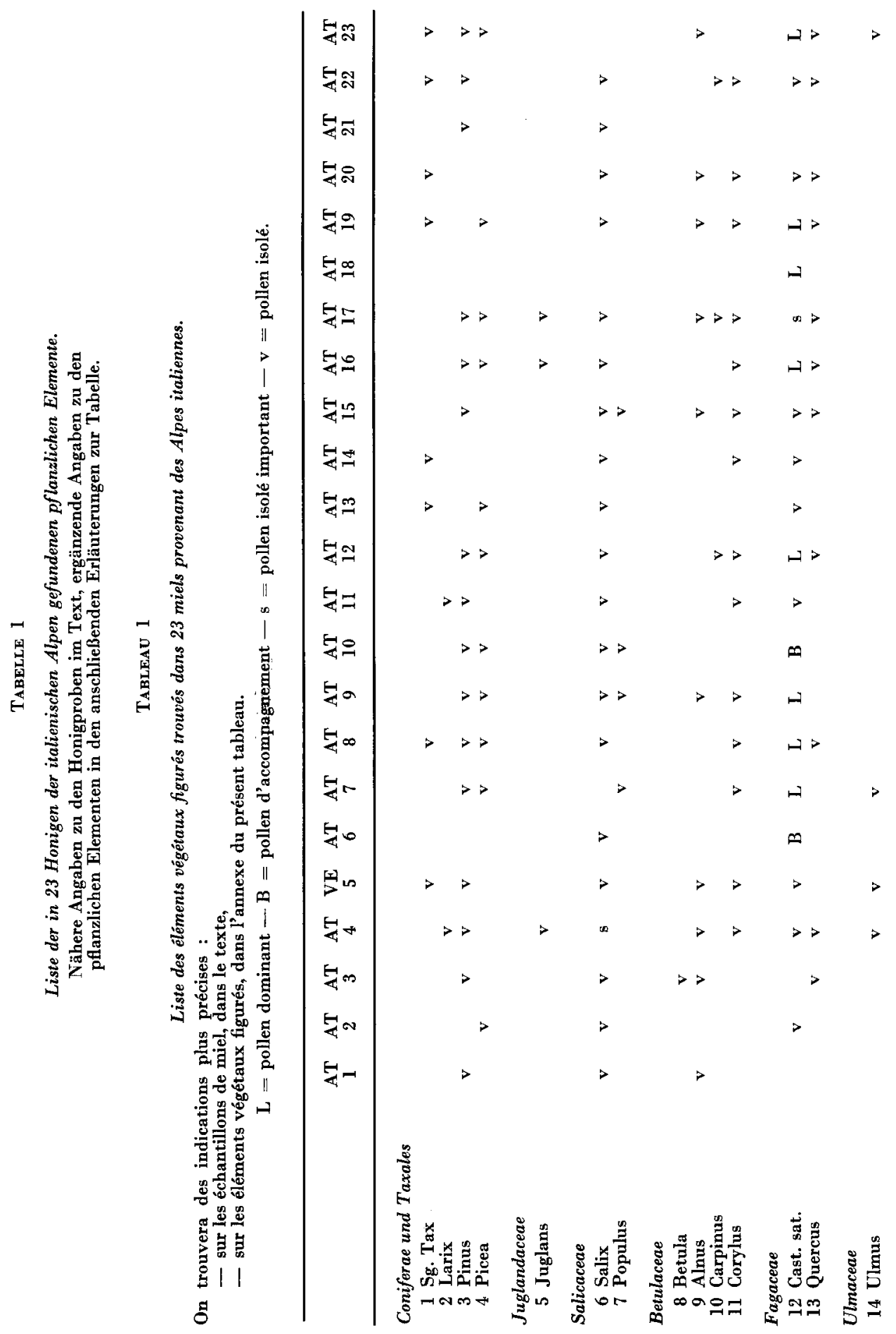




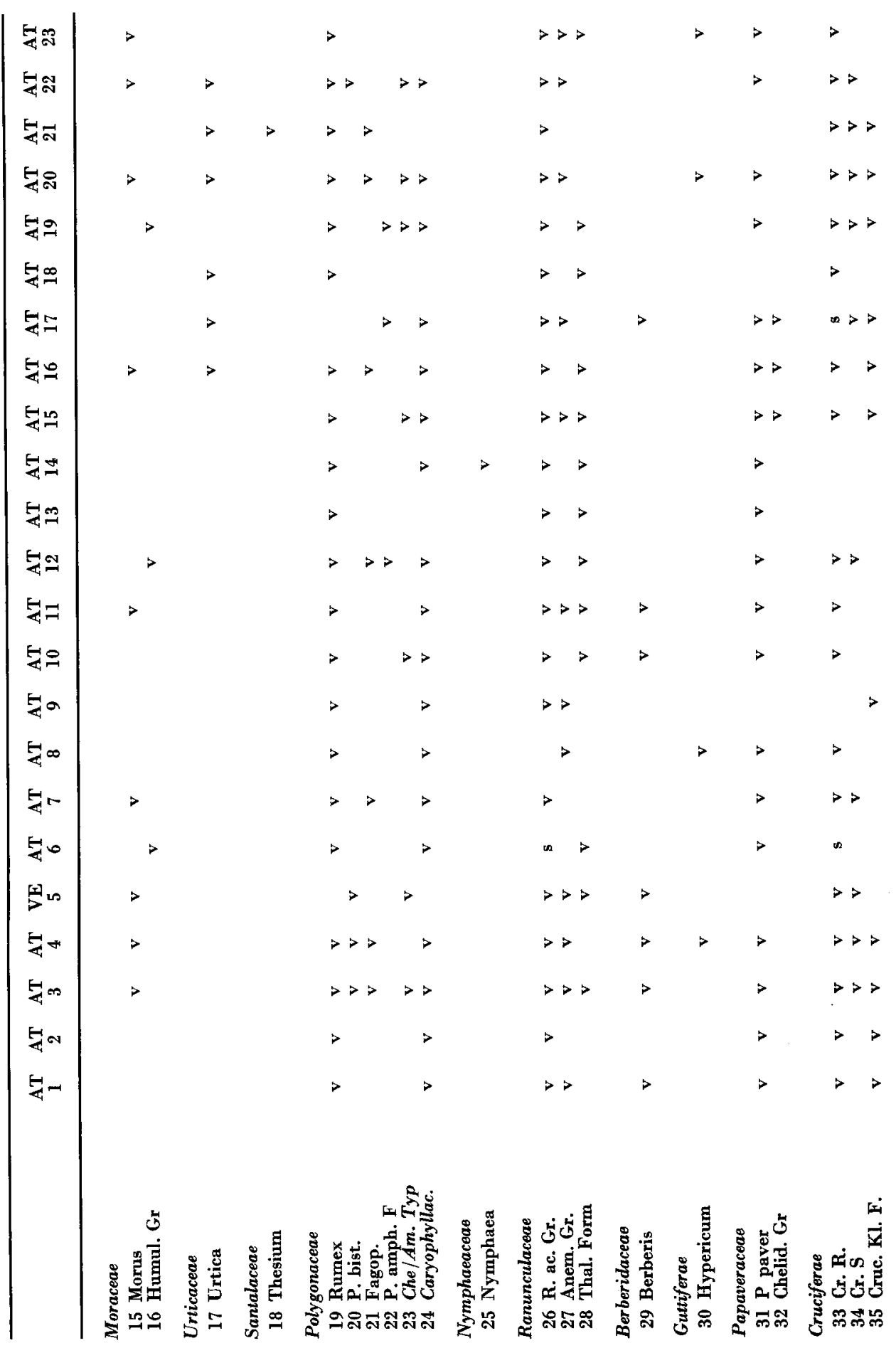


G. VORWOHL

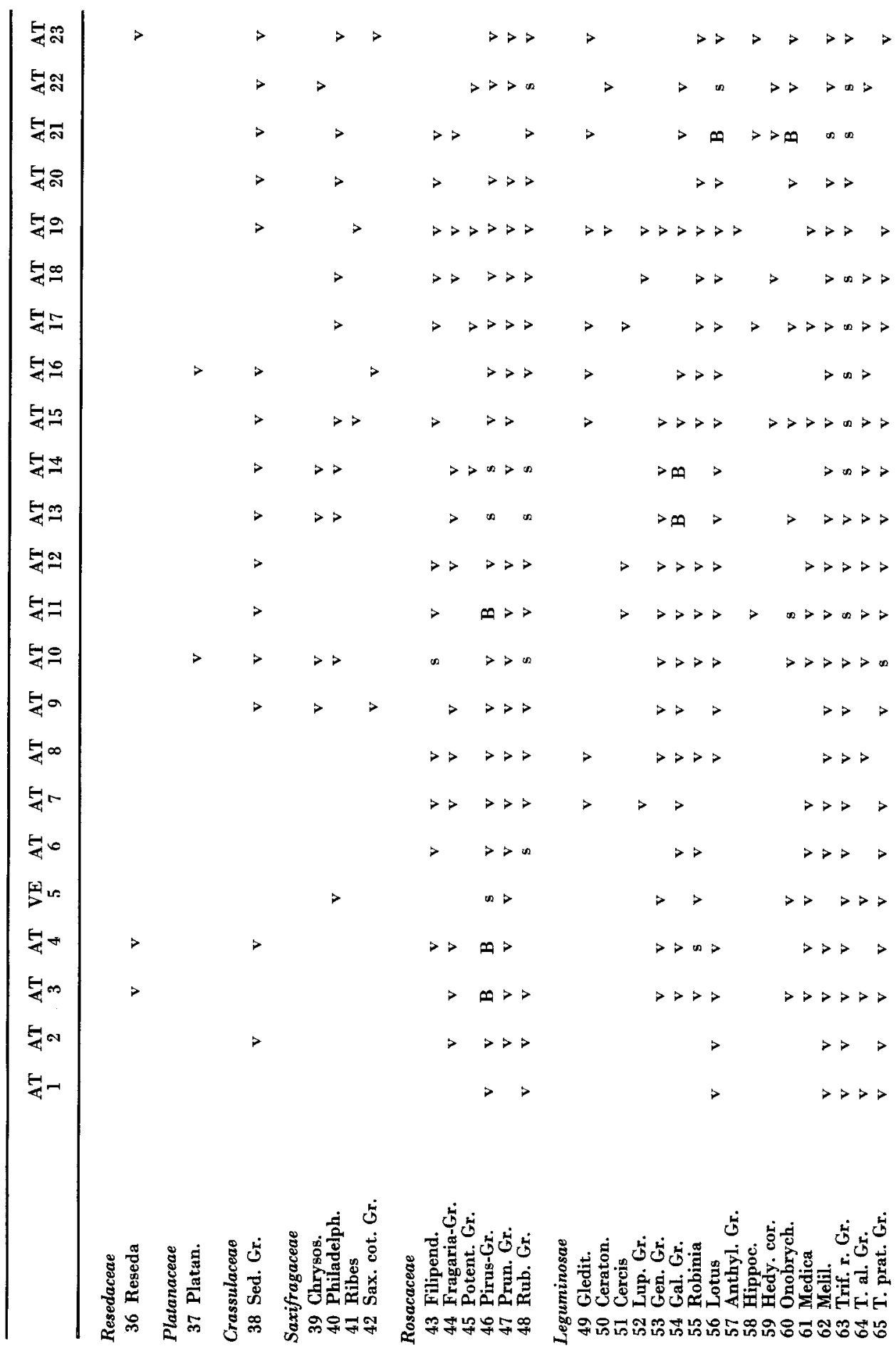




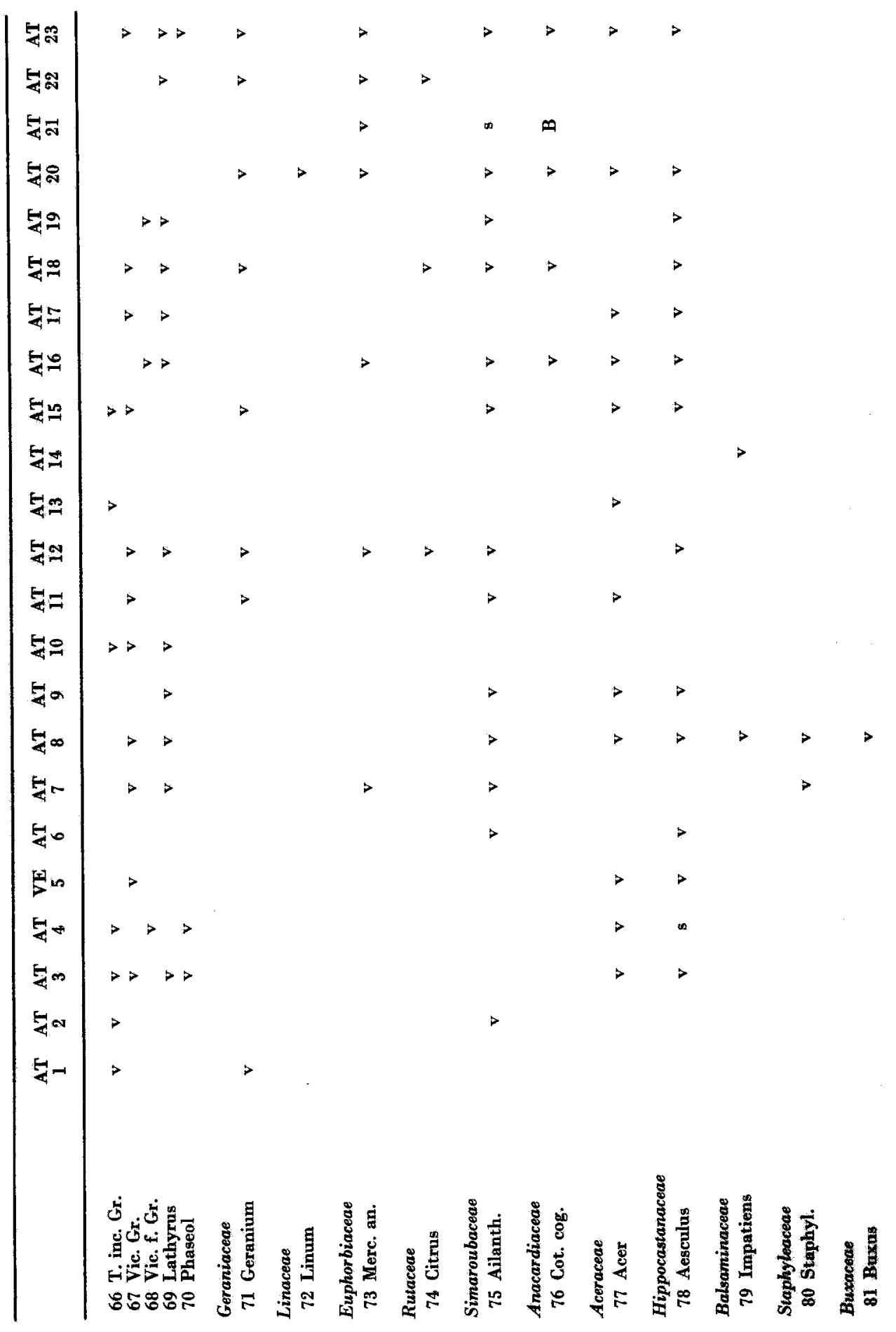




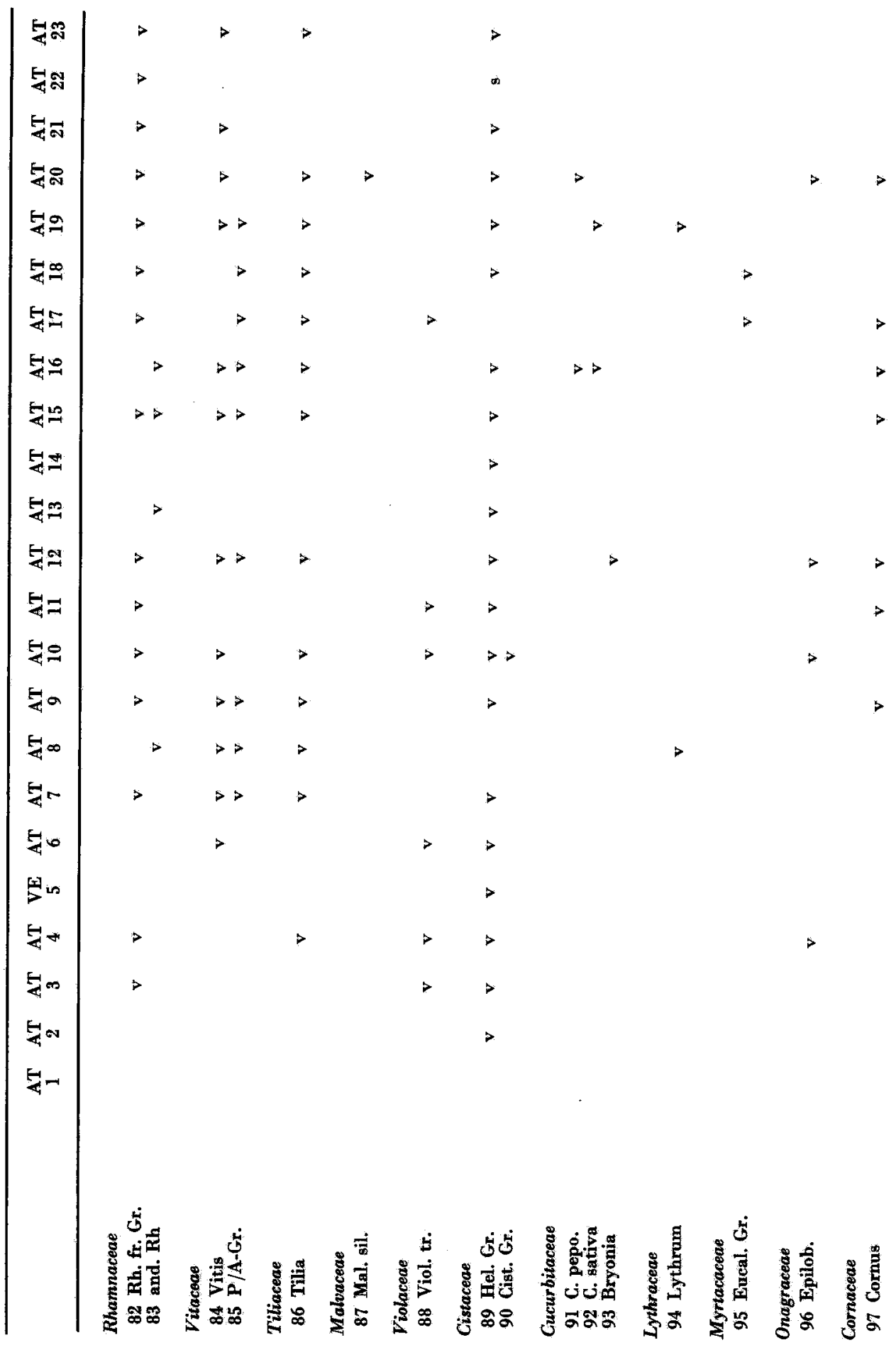




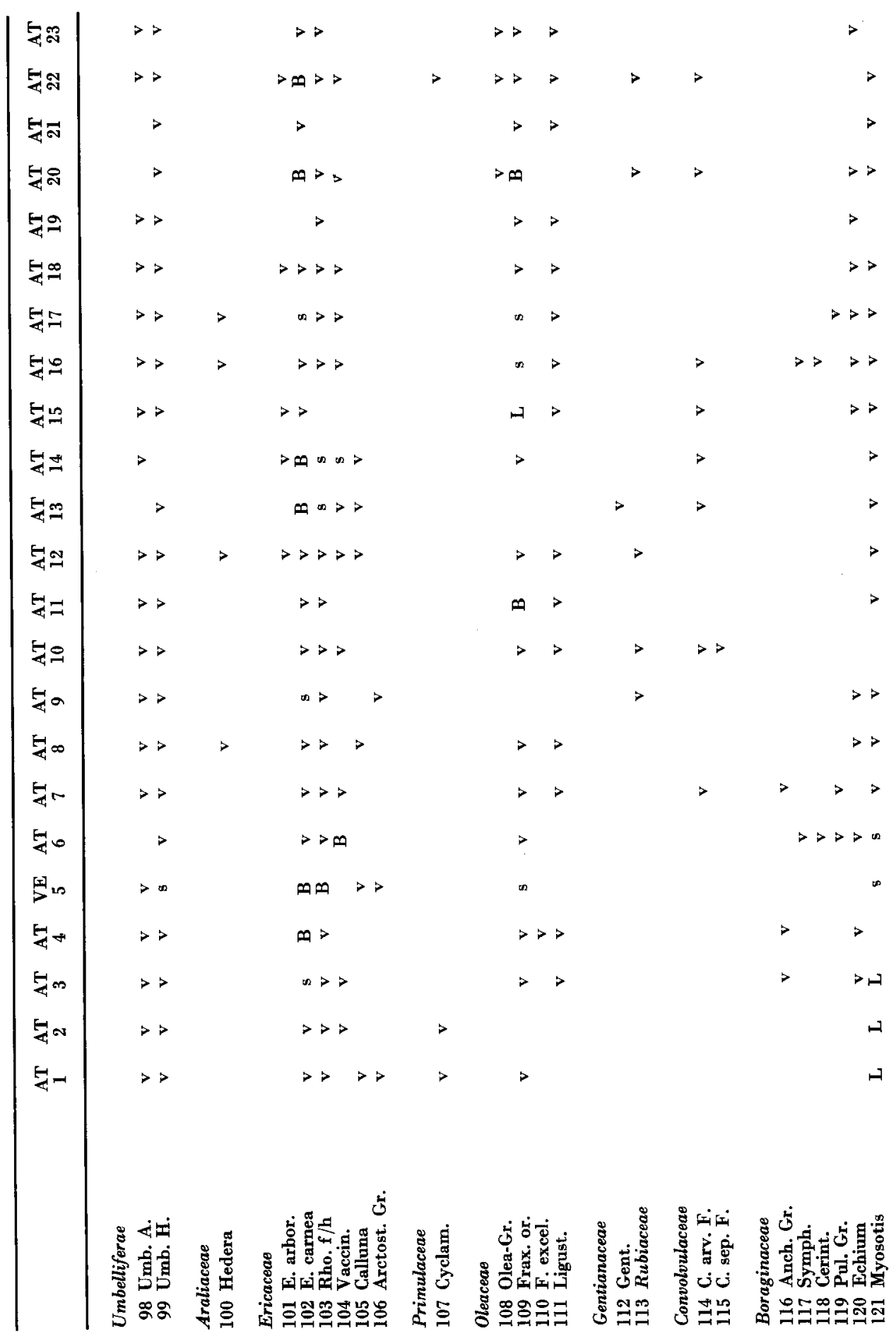




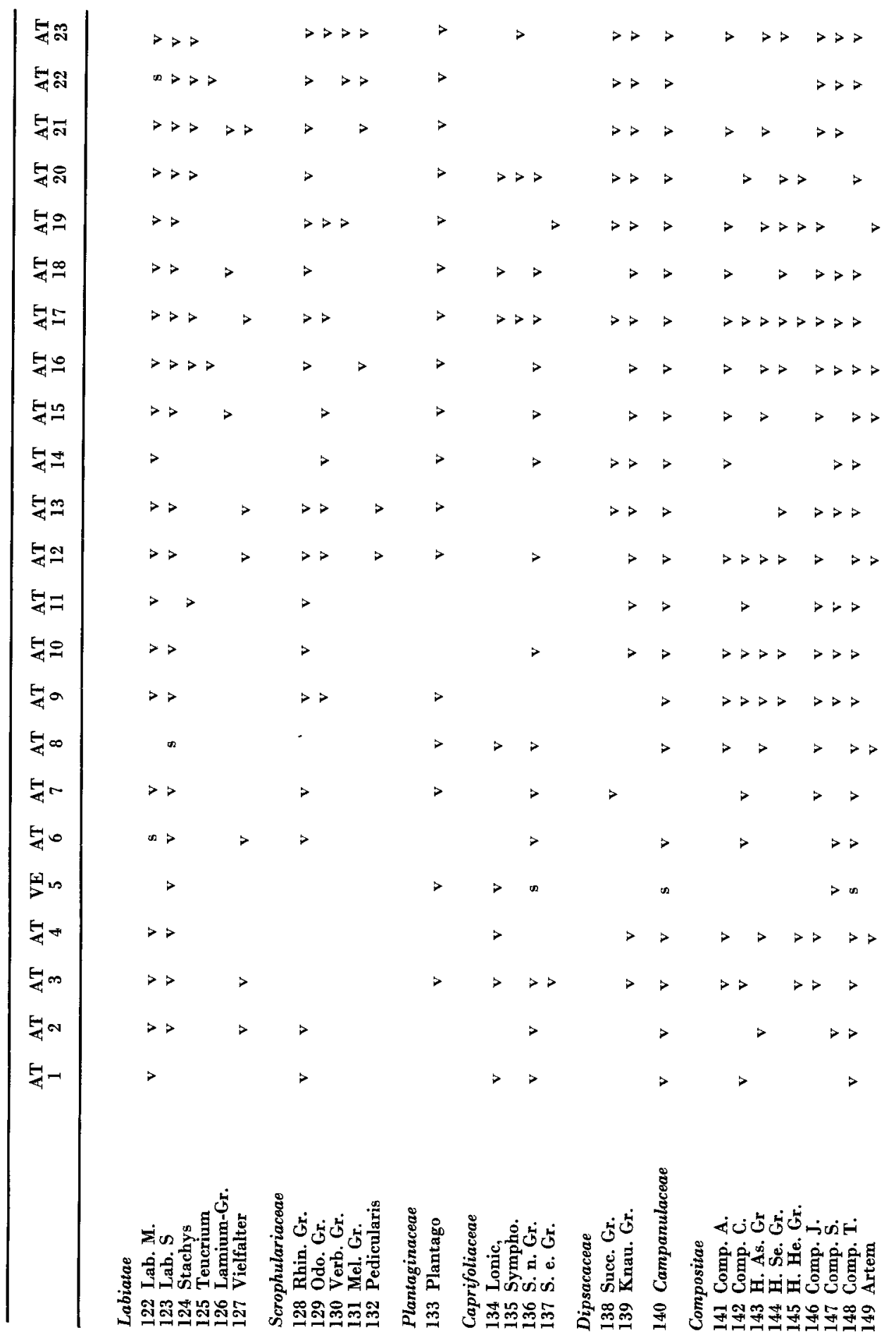




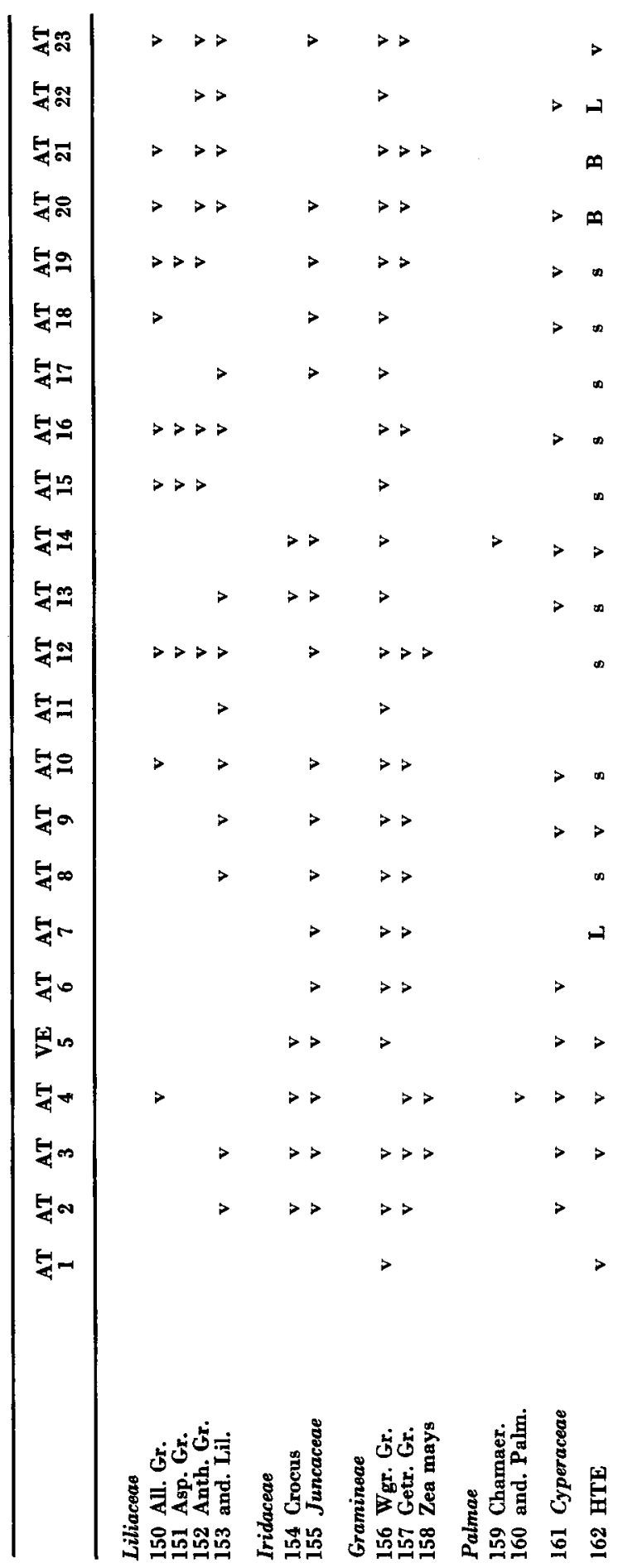




\section{ERLÄUterungen ZUR POLLENListe}

Die Erläuterungen umfassen die deutschen und italienischen Populärnamen, soweit sich Angaben in der Literatur finden, ferner, falls nötig, Bemerkungen zur Identifizierung und Benennung der Pollen. Literaturangaben zur Beschreibung der Pollenformen wurden nur gegeben, wenn sich die fragliche Form nicht in den melissopalynologischen Werken findet, die als Handbibliothek zur Verfügung stehen sollten : Allen, 1937, Armbruster u. JacoBs, 1934-1935, Ammbruster u. Oenike, 1929, Evenius u. Focke, 1967, Griebel, 1930-1931, Hodges, 1952, Hyde u. Adams, 1958, Louveaux, 1970, Maurizio u. Louveaux, 1965, ZANDER, 1935, 1937, 1941. L bedeutet Leitpollen, B Begleitpollen, swichtiger Einzelpollen, v Einzelpollen.

\section{Coniferae und Taxales}

1. Sammelgruppe : Taxus, Juniperus, Cupressus, Chamaecyparis etc. Eibe, Wacholder, Zypresse, Scheinzypresse, ital. : Tasso, Ginepro, Cipresso, Cipresso di Lawson. Wegen des selten und vereinzelten Auftretens lohnt in unserem Zusammenhang eine nähere Identifizierung nicht.

2. Larix, Lärche, ital. : Larice.

3. Pinus, Kiefer, ital. : Pino.

4. Picea, Fichte, ital. : Abete rosso.

Die genauere Bestimmung der Pinus- und Picea- Arten wurde nicht angestrebt.

\section{Juglandaceae}

5. Juglans, NuBbaum, ital. : Noce.

\section{Salicaceate}

6. Salix, Weide, ital. : Salice.

7. Populus, Pappel, ital. : Pioppo.

\section{Betulaceae}

8. Betula, Birke, ital. : Betulla.

9. Alnus, Erle, ital. : Ontano.

10. Carpinus, Hainbuche, ital. : Carpino.

11. Corylus, Haselnuß, ital. : Nocciolo.

\section{Fagaceae}

12. Castanea sativa, Edelkastanie, ital, : Castagno.

13. Quercus, Eiche, ital. : Quercia.

\section{Ulmaceae}

14. Ulmus, Ulme, ital. : Olmo.

\section{Moraccae}

15. Morus, Maulbeerbaum, ital. : Gelso.

16. Humulus, Hopfen, ital. : Luppolo.

\section{Urticaceae}

17. Urtica, Brennessel, ital. : Ortica. Sehr ähnlich auch Parietaria (Urticaceae) und Broussonetia papyrifera (Moraceae).

\section{Santalaceae}

18. Thesium, Bergflachs. Beschreibung ERdrman (1966), identifiziert nach der Sammlung M. Haddad, Beyrouth, Geographisches Institut der Université Libanaise (Acetolysiertes Material). Taf. II, Abb. 5. 


\section{Polygonaceae}

19. Rumex, Ampfer, ital. : Romice, Acetosa, Solegiola.

20. Polygonum bistorta, Wiesenknöterich, ital. : Bistorta, Serpentina.

21. Fagopyrum, Buchweizen, ital. : Grano saraceno.

22. Polygonum amphibium-Form (periporat).

23. Chenopodiaceae/Amaranthaceae-Typ (periporat).

24. Caryophyllaceae, ital. : Cariofillacee.

\section{Nymphaeaceae}

25. Nymphaea, Seerose, ital. : Ninfea.

\section{Ranunculaceae}

26. Ranunculus acer-Gruppe, Hahnenfuß-Gruppe, ital. : Ranunculo (gruppo di).

27. Anemone-Gruppe (tricolpat), Anemonen-Gruppe, ital. : Anemono (gruppo di).

28. Thalictrum-Form (periporat, z. B. Thalictrum, Anemone coronaria).

\section{Berberidaceae}

29. Berberis, Berberitze, ital. : Crespino.

\section{Guttiferae}

30. Hypericum, Johanniskraut, ital. : Arcidiavolo.

\section{Papaveraceae}

31. Papaver, Mohn, ital. : Papavero, Rosolaccio.

32. Chelidonium-Gruppe, Schöllkraut-Gruppe, ital. : Celidonia, Erba porrina (gruppo di).

\section{Cruciferae}

33. Cruciferae-R-Gruppe, namensgebende Art, Brassica napus napus, Raps, ital. : Colza, größter Durchmesser ca. $23 \mu$.

34. Cruciferae-S-Gruppe, namensgebende Art Sinapis arvensis, Senf, Ackersenf, ital. : Senapino, Senape dei campi, zwei Größen 30-35 $\mu$ und 23-26 $\mu$.

35. Cruciferen-Kleinformen (größter Durchmesser maximal $19 \mu$ ).

\section{Resedaceae}

36. Reseda, Reseda, ital. : Erba guada, Guaderella crociata et oltre specie di Reseda.

\section{Platanaceae}

37. Platanus, Platane, ital. : Platano.

\section{Crassulaceae}

38. Sedum-Gruppe, Fetthenne-Gruppe, ital. : Erba pignola.

\section{Saxifragaceae}

39. Chrysosplenium, Milzkraut, ital. : Crisosplenio.

40. Philadelphus, Falscher Jasmin, Pfeifenstrauch, ital. : Fiore d'Angiolo.

41. Ribes, Stachelbeere, Johannisbeere, ital. : Uva spina, Ribes.

42. Saxifraga cotyledon-Gruppe, Steinbrech-Gruppe, ital. : Sannicola (striate, tricolpate PK mit vielen Exineresten auf den Falten, die sich zu deckelartigen Gebilden zusammenschließen).

\section{Rosaceae}

43. Filipendula, Mädesüß, ital. : Filipendola.

44. Fragaria-Gruppe, Erdbeer-Gruppe, ital. : Fragola (gruppo di), Die Pollen sind manchmal schwer gegen die gelben striaten Pollen einiger Saxifraga-Arten abzugrenzen.

45. Potentilla-Gruppe, Fingerkraut-Gruppe, ital. : Potentilla (gruppo di).

46. Pirus-Gruppe, Kernobst-Gruppe, ital. : Pomacee.

47. Prunus-Gruppe, Steinobst-Gruppe, ital. : Drupacee.

48. Rubus-Gruppe, Himbeer-/Brombeer-Gruppe, ital. : Lampone, Rovo (gruppo di). 


\section{Leguminosae}

49. Gleditsia, Gleditschia, Christusdorn, ital. : Spina Christi.

50. Ceratonia siliqua, Johannisbrotbaum, ital. : Carrubo. In der "Handliteratur " findet sich nur eine wenig zureichende Beschreibung und Abbildung bei Armbruster und JacoBs 1934/35. Der Pollen wurde daher beschrieben und abgebildet (Taf. I, Abb. la _- If), siehe auch HoRowitz und BAuM 1967.

51. Cercis siliquastrum, Judasbaum, ital. Albero di Giuda. Pollenbeschreibung : Taf. II, Abb. 3a - 3c, siehe auch Horowitz und Baum 1967.

52. Lupinus-Gruppe, ital. Lupino.

53. Genista-Gruppe, Sammelgruppe aus den Pollen der Gattungen Cytisus (Geißklee, ital. : Citiso), Genista (Ginster, ital. Ginestra), Sarothamnus (Besenginster, ital. Ginestra de carbonai), Laburnum (Goldregen, ital. Maggiocicondolo), Ulex (Stechginster, ital. Ginestrone).

54. Galega-Gruppe, Geißrauten-Gruppe, ital. : Crapaggine (gruppo di). Vom Geißrautenpollen schwer zu unterscheiden ist der Pollen von Sophora, Schnurbaum, ital. : Sofora.

55. Robinia, Akazie oder falsche Akazie, Robinie, ital. : Acacia, Robinia.

56. Lotus, Hornklee, ital. Trifoglio giallo, sehr ähnlich auch die Pollen von Coronilla scorpioides, Kronwicke, ital. : Canterella.

57. Anthyllis, Wundklee, ital. : Vulneraria.

58. Hippocrepis, Hufeisenklee, ital. : Sferra Cavallo.

59. Hedysarum coronarium, Süßklee, Italienischer Hahnenkopf, ital. : Sulla. Bei vereinzeltem Auftreten ist eine Verwechslung mit den Pollen einiger Astragalus-Arten denkbar, z.B. Astragalus falcatus und Astragalus monspessulensis. Im Falle größerer Häufigkeit dürfte die Bestimmung bis zur Art unproblematisch sein.

60. Onobrychis, Esparsette, ital. : Lupinella.

61. Medicago, Luzerne, ital. : Medica.

62. Melilotus, Honigklee, Steinklee, ital. : Vetturina.

63. Trifolium repens-Gruppe, Weißklee-Gruppe, ital. : Trifoglio bianco (gruppo di), in diese werden hier zusammengefaßt : $T$. repens, $T$. hybridum, T. arvense, $T$. resupinatum, möglicherweise auch noch andere Trifolium-Arten, denn die zahlreichen Species der Gattung sind palynologisch noch nicht ausreichend untersucht. Auch Pollen anderer Leguminosengattungen können mit Weißklee verwechselt werden, z. B. Dorcynium ornithopus und Astragalus glycophyllos, zumal wenn sie vereinzelt vorkommen und ungünstig im Präparat liegen.

64. Trifolium alexandrinum-Gruppe, Alexandrinerklee-Gruppe, ital. : Trifoglio alexandrino, Bersim. Der Alexandrinerklee liefert den kleinsten Pollen in der Reihe der Rotkleeähnlichen Pollen nach Zander $33: 31,8 \mu$ (in meinem Vergleichspräparat etwas kleiner). Es kann also zu Überschneidungen mit kleinen Pollen von Trifolium pratense und $T$. alpestre kommen. Die "Alexandrinerklee " Befunde in höheren Lagen sind wahrscheinlich meist so zu interpretieren.

65. Trifolium pratense-Gruppe, Rotklee-Gruppe, ital. : Trifoglio violetto, Trifoglio commune (gruppo di). Neben dem Rotklee fallen in diese Gruppe auch Trifolium stellatum und $T$. angustifolium, wahrscheinlich auch noch andere Arten.

66. Trifolium incarnatum-Gruppe, Inkarnatklee-Gruppe, ital. : Trifoglio incarnato (gruppo di). Größter Pollen der Rotklee-Reihe. Die größten Pollen des Rotklees und der ähnlichen Arten entsprechen kleinen Inkarnatklee-Pollen.

67. Vicia-Gruppe, Wicken-Gruppe, ital. : Veccia (gruppo di). Die Gruppe umfaßt die kleinen Wickenpollen (gröBter Durchmesser bis zu $37 \mu$ ) mit glatter oder fast glatter Exine.

68. Vicia faba-Gruppe, Pferdebohnen-Gruppe, ital. : Fava (gruppo di). Neben Vicia faba gehört $V$. lutea hierhin.

69. Lathyrus, Platterbsen, ital. : Cicerchia, Fior-galletto.

70. Phaseolus, Stangenbohne, Buschbohne, ital. : Fagiuolo, Gagiolo.

\section{Geraniaceae}

71. Geranium, Storchschnabel, ital. : Geranio.

\section{Linaceae}

72. Linum, Lein, ital. : Lino.

\section{Euphorbiaceae}

73. Mercurialis, Bingelkraut, ital. : Mercorella 


\section{Rutaceae}

74. Citrus, Agrumen, Zitrone, Apfelsine usw., ital. : Agrumi, Limone, Arancio ecc. Die Pollen der verschiedenen Citrus-Arten und-Sorten wurden noch nicht eingehend bearbeitet, eine Unterscheidung dürfte auf jeden Fall schwierig sein.

\section{Simaroubaceae}

75. Ailanthus, Götterbaum, ital. : Ailanto.

\section{Anacardiaceae}

76. Cotinus coggygria, Perückenstrauch, ital. : Scotano, Cotino, Capecchio. Der Pollen ist nur bei Armbruster u. Oenike unzureichend abgebildet und beschrieben. Näheres siehe Taf. II Abb. $4 a-4 f$.

\section{Aceraceae}

77. Acer, Ahorn, ital. : Acero.

\section{Hippocastanaceae}

78. Aesculus, Roßkastanie, ital. : Ippocastano, Castagno d'India.

\section{Balsaminaceae}

79. Impatiens, Springkraut, ital. : Noli me tangere.

\section{Staphyleaceae}

80. Staphylea, Pimpernuß, ital. : Borsolo, Lacrime di Giobbe.

\section{Buxaceae}

81. Buxus, Buchsbaum, ital. : Bosso, Bossolo.

\section{Rhamnaceae}

82. Rhamnus frangula, Faulbaum-Gruppe, ital. : Frangola, Alno nero (gruppo di).

83. Rhamnaceen, andere, Farbe und Exineskulptur abweichend von Rhamnus frangula, nicht näher bestimmt.

\section{Vitaceae}

84. Vitis, Weinrebe, ital. : Vite.

85. Parthenocissus /Ampelopsis-Gruppe, wilder Wein, ital. : Vite del Canada.

\section{Tiliaceae}

86. Tilia, Linde, ital. : Tiglio.

\section{Malvaceae}

87. Malva silvestris-Gruppe, wilde Malde (Gruppe), ital. : Malva (gruppo di).

\section{Violaceae}

88. Viola tricolor-Gruppe, Stiefmütterchen-Gruppe, ital. : Viola del pensiero (gruppo di).

\section{Cistaceae}

89. Helianthemum-Gruppe, Sonnenröschen, ital. : Eliantemo (gruppo di), (umfaßt Helianthemum u. die Cistus spec. mit fein- Helianthemum-ähnlich reticuliertem Pollen).

90. Cistus, Zistrosen, ital. : Cisto, (Cistaceen-Pollen mit weitmaschigem Reticulum).

\section{Cucurbitaceae}

91. Cucurbita pepo, Kürbis (mit allen Varietäten), ital. : Zucche (molteplici varietà).

92. Cucumis sativus, Gurke, ital. : Cetriolo.

93. Bryonia, Zaunrübe, ital. : Fescera, Vite bianca, Brionia. 


\section{Lythraceae}

94. Lythrum, Weiderich, ital. : Salcerella, Riparella.

\section{Myrtaceae}

95. Eucalyptus-Gruppe, ital. : Eucalitto (gruppo di).

\section{Onagraceae}

96. Epilobium/Chamaenerion, Weidenröschen, ital. : Camenerio, Epilobio.

\section{Cornaceae}

97. Cornus alba/C. sanguinea, Hartriegel, ital. : Sanguinella.

\section{Umbelliferae}

98. Umbellifere A, namensgebende Art Anthriscus, ital. : Cerfoglio.

99. Umbellifere $\mathbf{H}$, namensgebende Art Heracleum, ital. : Panace.

\section{Araliaceae}

100. Hedera helix, Efeu, ital. : Edera, Ellera.

\section{Ericaceae}

101. Erica arborea, Baumheide, ital. : Scopo.

102. Erica carnea, Schneeheide, ital. : Scopina.

103. Rhododendron ferrugineum / Rh. hirsutum, Alpenrose, ital. : Rosa delle Alpi, Rododendro.

104. Vaccinium, Heidelbeere (u.a.), ital. : Mirtillo rosso e nero (e altri).

105. Calluna vulgaris, Besenheide, ital. : Brentoli.

106. Arctostaphylos-Gruppe, Bärentraube-Gruppe, ital, : Uva orsina (gruppo di).

\section{Primulaceae}

107. Cyclamen-Gruppe, Alpenveilchen-Gruppe, ital. : Ciclamino (gruppo di). Nur bei ArmBRUSTER u. JAKOBS eine sehr einfache Zeichnung.

\section{Oleaceae}

108. Olea-Gruppe, Ölbaum u. Oleaster-Gruppe, ital. : Olivo, Oleastro (gruppo di).

109. Fraxinus ornus-Gruppe, Schmuckeschen-Gruppe, ital. : Orniello (gruppo di). Olea u. Fraxinus ornus sind im Einzelfall manchmal sehwer zu unterscheiden. Hierher gehört auch Phillyrea.

110. Fraxinus excelsior, Esche, ital. : Frassino.

111. Ligustrum, Liguster, Rainweide, ital. : Ligustro.

\section{Gentianaceae}

112. Gentiana, Enzian, ital. : Genziana.

\section{Rubiaceae}

113. Galium, Labkraut, Klebkraut, ital. : Caglio, Ataccaveste, Asperula, Waldmeister. ital.: Stellina odorosa.

\section{Convolvulaceae}

114. Convolvulus arvense-From (tricolpat), Acker-Winden-Form, ital. : Vilucchio (forma di). 115. Convolvulus sepium-Form (periporat, nicht echinat), Zaun-Winde, ital. : Vilucchione (forma di).

\section{Boraginaceae}

116. Anchusa-Gruppe, Ochsenzungen-Gruppe, ital. : Lingua di bue (gruppo di).

117. Symphytum-Gruppe, Beinwell-Gruppe, ital. : Consolida, Simfito (gruppo di).

118. Cerinthe minor-Gruppe, kleine Wachsblume (Gruppe), ital. : Erba tortora (gruppo di). Beschreibung bei Hazscinski, 1952, Fossec u. F. Rutrner, 1966 und VorwohL, 1968.

119. Pulmonaria-Gruppe, Lungenkraut-Gruppe, ital. : Polmonaria (gruppo di).

120. Echium, Natterkopt, ital. : Erba viperina.

121. Myosotis, Vergißmeinnicht, ital. : Non-ti-Scordar-di-me. 
Labiatae

122. Labiatae M (Majorana-Form, Majoran-Form, ital. : Forma di Maggiorana).

123. Labiatae S (Salvia-Form, Salbei-Form, ital. : Forma di Salvia).

124. Stachys-Gruppe, Wusperkraut-Gruppe, ital. : Stachis, Vettonica (gruppo di).

125. Teucrium, Gamander, ital. : Calamandrea, Bugola.

126. Lamium-Gruppe, Taubnessel-Gruppe, ital. : Dolcimele (gruppo di).

127. Vielfalter, Labiatae-Pollen mit mehr als 6 , meist 8 Keimstellen, Bei Satureja hortensis z. B. kommen neben 6-colpaten in hohen Prozentsätzen 8-faltige PK vor (S. hortensis = Bohnenkraut, ital. : Santoreggia).

Scrophulariaceae

128. Rhinantus-Gruppe, Klappertopf-Gruppe, ital. : Cresta di gallo.

129. Odontites-Gruppe, Zahntrost-Gruppe.

130. Verbascum-Gruppe, Königskerzen-Gruppe, ital. : Verbasco, Tasso barbasso.

131. Melampyrum-Gruppe, Wachtelweizen, ital. : Melampiro.

132. Pedicularis comosa-Gruppe (2-syncolpat), Läusekraut, ital. : Pedicolaria. Beschreibung bei BEUg, 1961, s. auch Hegr, 1969 für acetolysiertes Material.

\section{Plantaginaceae}

133. Plantago, Wegerich, ital. : Centoneroi, Piantagine, Lanciuola, Mestolaccio.

\section{Caprifoliaceae}

134. Lonicera, Heckenkirsche, ital. : Caprifoglio.

135. Symphoricarpus, Schneebeere.

136. Sambucus nigra-Gruppe, Schwarzer Holunder (Gruppe), ital. : Sambuco (gruppo di).

137. Sambucus ebulus, Zwergholunder, ital. : Ebbio, Lebbio, Colore.

\section{Dipsacaceae}

138. Succisa-Gruppe, Teufelsabbiß-Gruppe, ital. : Morso del diavolo (gruppo di).

139. Knautia-Gruppe, Witwenblume, ital. : Vedovina campestre, Ambretta, Gallinacia, Suocere (gruppo di).

140. Campanulaceae

Compositae

141. Compositae A-Form (namensgebende Art Achillea, Schafgarbe, ital. : Achillea, Millefoglio).

142. Compositae C (namensgebende Art Centaurea cyanus, Kornblume, ital. : Fioraliso).

Compositae H-Form

143. Aster-Gruppe, Aster-Gruppe, ital. : Astro (gruppo di).

144. Senecio-Gruppe, Greiskraut-Gruppe, ital. : Senecione, Verzettina (gruppo di).

145. Helianthus-Gruppe, Sonnenblumen-Gruppe, ital. : Girasole (gruppo di).

146. Compositae J-Form (namensgebende Art Centaurea jacea, Flockenblume, ital. : Steccione, (gruppo di).

147. Compositae S (namensgebende Art Serratula, Scharte, ital. : Seratola).

148. Compositae T-Form (namensgebende Art Taraxacum, Löwenzahn, ital. : Tarassaco).

149. Artemisia-Gruppe, BeifuB-Gruppe, ital. : Assenzio, Amarella.

\section{Liliaceae}

150. Allium-Gruppe, Lauch-Gruppe, ital. : Aglio (gruppo di).

151. Asparagus-Gruppe, Spargel-Gruppe, ital. : Asparago (gruppo di).

152. Anthericum-Gruppe, Graslilien-Gruppe, ital. : Lilioafodello (gruppo di), (s. auch Beschreibung u. Abb. bei Beug, 1961).

153. andere Liliaceen-Pollen

\section{Iridaceae}

154. Crocus, Krokus, ital. : Zafferano selvatico.

155. Juncaceae, ital. : Giuncacee. 
Gramineae, ital. : Graminacee.

156. Wildgras-Gruppe (größter Pollendurchmesser $37 \mu$, s. Beug, 1961).

157. Getreide-Gruppe (größter Pollendurchmesser über $37 \mu$, außer Zea mays).

158. Zea mays (über $75 \mu$ ), ital. : Gramturco.

\section{Palmae}

159. Chamaerops, Zwergpalme, ital. : Palma, (Beschreibungen auch bei Beug, 1961 u. ErdTMAN, 1966).

160. andere Palmenpollen (monocolpat).

161. Cyperaceae, ital. : Cyperacee.

162. Honigtauelemente (Pilzsporen und Hyphen, Algenzellen u. Algenverbände, Wachselemente der Honigtau-ausscheidenden Insekten. Die Häufigkeitsangaben bedeuten hier : $\mathbf{L}=$ sehr viel, $\mathbf{B}=$ viel, $\mathbf{s}=$ mittlere Menge, $\mathbf{v}=$ wenig. 